\title{
An architectural hypothesis for direction selectivity in the visual cortex: the role of spatially asymmetric intracortical inhibition
}

\author{
Silvio P. Sabatini, Fabio Solari \\ Department of Biophysical and Electronic Engineering, University of Genoa, Via all'Opera Pia 11/A, I-16145 Genoa, Italy \\ Received: 8 May 1998 / Accepted in revised form: 10 November 1998
}

\begin{abstract}
Within a linear field approach, an architectural model for simple cell direction selectivity in the visual cortex is proposed. The origin of direction selectivity is related to recurrent intracortical interactions with a spatially asymmetric character along the axis of stimulus motion. No explicit asymmetric temporal mechanisms are introduced or adopted. The analytical investigation of network behavior, carried out under the assumption of a linear superposition of geniculate and intracortical contributions, shows that motion sensitivity of the resulting receptive fields emerges as a dynamic property of the cortical network without any feed-forward direction selectivity bias. A detailed analysis of the effects of the architectural characteristics of the cortical network on directionality and velocityresponse curves was conducted by systematically varying the model's parameters.
\end{abstract}

\section{Introduction}

Recent theoretical and neurophysiological studies (DeAngelis et al. 1993a,b; Hamilton et al. 1989; McLean et al. 1994; Reid et al. 1991; Tolhurst and Dean 1991) pointed out that the origin of direction selectivity can be related to the linear space-time receptive field structure of simple cells. A large class of simple cells shows a very specific space-time behavior in which the spatial phase of the receptive field changes gradually as a function of time. This results in receptive field profiles that tilt along an oblique axis in the space-time domain (i.e., they are space-time inseparable). Accurate estimates of the velocity components to which the cell is selective (the preferred speed of motion) can be derived by measuring the slope of oriented receptive field subregions in the space-time domain. Since lateral geniculate nucleus

Correspondence to: S.P. Sabatini

(e-mail: silvio@dibe.unige.it,

Tel.: + 39-10-3532289, Fax: + 39-10-3532795)
(LGN) cells do not exhibit tilted subregions, the origin of space-time inseparability must take place within the striate cortex. In general, the construction of inseparable simple-cell receptive fields implies a position-dependent alteration of the temporal response characteristics of the afferent inputs, presumably associated with cortical circuits characterized by asymmetric architectural schemes in space and/or time (for a review, see Koch and Hildreth 1987). Several models have been proposed. Some postulate the combination of spatially offset geniculate receptive fields with different temporal dynamics (Mastronarde 1987; Saul and Humphrey 1990; Wimbauer et al. 1994, 1997). Others assume intracortical interactions among separable simple-cell receptive fields, possibly mediated by cortical interneurons (Ganz 1984; Ruff et al. 1987; Sillito 1977; Somers et al. 1995). Most models, however, do not consider explicit recurrent asymmetric intracortical processes, and furthermore, a clear distinction between the roles of purely spatial and purely temporal mechanisms has not yet been made.

In this paper, we point out that a purely spatial asymmetry is sufficient to generate directional selectivity when spatially asymmetric contributions arise through recurrent intracortical inhibitory circuits. Therefore, the space-time orientation of the resulting receptive fields emerges as a dynamic property of our cortical network, thus leading to highly structured space-time receptive fields from space-time separable Gaussian subunits (i.e., LGN inputs). The model starts from a simple basic arrangement of inhibitory interconnections characterized by a symmetrical spatial structure. By adding different degrees of asymmetry, different types of direction selectivities arise, like those observed in the visual cortex.

\section{The architectural model}

\subsection{Basic architecture}

Following a linear neural field approach (Amari 1977; Wilson and Cowan 1972), we regard the visual cortex as a continuous distribution of neurons and synapses. 
Accordingly, the geniculocortical pathway is modeled by a multilayer network coupled through feedforward and feedback connections, both inter- and intralayers (Mallot and Giannakopoulos 1996). The network layers represent spatially homogeneous population models where each point in space $[\mathbf{x}=(x, y)]$ corresponds to an 'average neuron.' The spatial coupling of these populations is described continuously by space-invariant kernels on the plane; the kernels represent the functional spread of the synaptic influence of a population on its neighbors, as mediated by local axonal and dendritic fields.

An architectural diagram of the cortical network is shown in Fig. 1. Two populations of cortical cells can be distinguished: excitatory cells and inhibitory interneurons. To each population corresponds a neural layer that is activated by retinotopic LGN inputs. The dynamics of each layer is modeled as a first-order low-pass filter -characterized by a specific time constant.

Recurrent processing depends upon the interactions between the excitatory and inhibitory populations (P-S system). If one assumes identical time constants and identical LGN inputs to both populations, the resulting excitation $e(\mathbf{x}, t)$ can be obtained as the solution of a single-layer linear field equation of lateral inhibition type (Amari 1977):

$$
\begin{aligned}
\tau \frac{d e(\mathbf{x}, t)}{d t}= & -e(\mathbf{x}, t)+b \int k_{\mathrm{d}}(\mathbf{x}-\xi) m(\xi, t) d \xi \\
& +\int k_{\mathrm{d}}(\mathbf{x}-\xi) e_{0}(\xi, t) d \xi
\end{aligned}
$$

with

$m(\mathbf{x}, t)=\int k_{\mathrm{a}}(\mathbf{x}-\xi) e(\xi) d \xi$

where $b$ is the interaction strength, $k_{\mathrm{a}}(\mathbf{x}-\xi)$ and $k_{\mathrm{d}}(\mathbf{x}-\xi)$ are the spatial distributions of the pre- and post-synaptic sites, respectively, modeled by Gaussian connection density functions; $m(\xi, t)$ is the distribution of presynaptic activity where excitatory and inhibitory contributions accumulate before being collected by the recurrent loop or by further processing stages.
The LGN contribution $\int k_{\mathrm{d}}(\mathbf{x}-\xi) e_{0}(\xi, t) d \xi$ can be modeled directly as a spatiotemporal convolution on the visual input $s(\mathbf{x}, t)$ through a separable kernel, $h_{0}(\mathbf{x}, t)=f_{0}(\mathbf{x}) g_{0}(t)$, characterized in the spatial domain by a Gaussian shape with an extension $\sigma_{0}$ and, in the temporal domain, by the biphasic impulse response:

$g_{0}(t)= \begin{cases}A\left[t / \alpha_{1}^{2} \exp \left(-t / \alpha_{1}\right)\right. & \\ \left.-L t / \alpha_{2}^{2} \exp \left(-t / \alpha_{2}\right)\right] & \text { for } t \geq 0 \\ 0 & \text { for } t<0\end{cases}$

where $\alpha_{1}=8 \mathrm{~ms}, \alpha_{2}=16 \mathrm{~ms}, L=0.9$ (Maex and Orban 1992) and $A$ is a normalization factor. The asymptotic behavior of the filter in (3) can be compared with that of a first-order low-pass filter with an equivalent time integration constant $\tau_{0} \simeq 20 \mathrm{~ms}$. On such a basis, the geniculate (i.e., feedforward) and the intracortical (i.e., feedback) dynamics can be compared directly through the time constants $\tau_{0}$ and $\tau$.

\subsection{Average intracortical connectivity}

The spatial distribution of inhibitory and excitatory influences can be summarized in the feedback interaction kernel defined as

$k_{\mathrm{FB}}(\mathbf{x}-\xi)=\int k_{\mathrm{d}}\left(\mathbf{x}-\xi^{\prime}\right) k_{\mathrm{a}}\left(\xi^{\prime}-\xi\right) d \xi^{\prime}$

Though $k_{\mathrm{FB}}$ may be positive and negative in sign, depending on excitatory and inhibitory couplings, in the following we shall principally consider negative kernels representative of merely inhibitory schemes. Comments on the role of additional recurrent excitation will be made in Sect. 3.6.

In a previous work (Sabatini 1996) we showed how the steady-state solutions of (1) and (2) can give rise to highly structured Gabor-like receptive field profiles, when inhibition arises from laterally distributed clusters of cells. The inhibitory kernel $k_{\mathrm{FB}}$ was modeled as the sum of two Gaussian functions, symmetrically offset with respect to the target cell (see Fig. 2): a

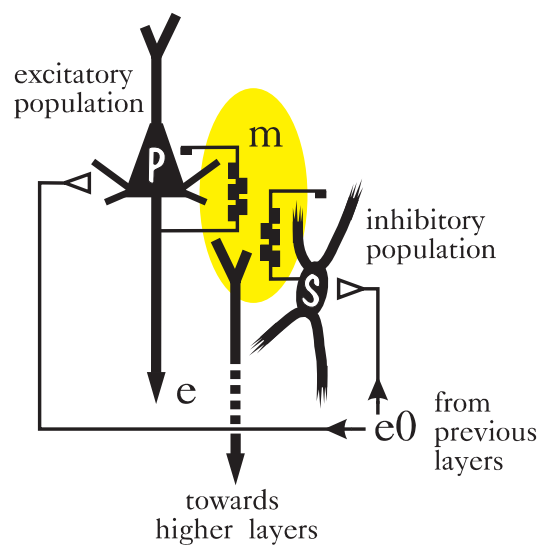

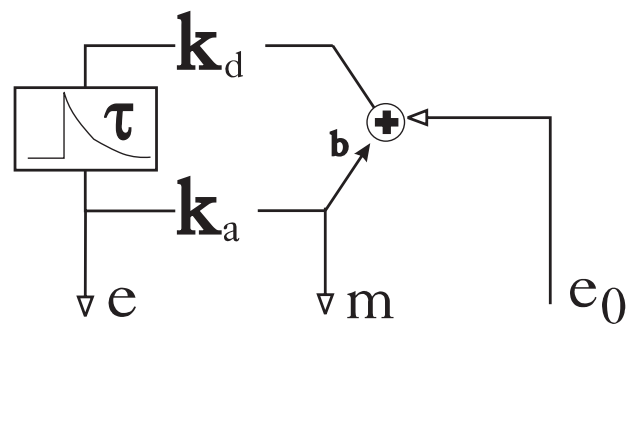

Fig. 1. a Schematic neural circuitry of our architecture: $P$ represents a population of excitatory neurons (putative pyramidal or spiny stellate cells), and $S$ a population of inhibitory interneurons (putative smooth or basket cells). Shaded region represents the presynaptic recurrent activity $m(\mathbf{x}, t)$ that is collected by the recurrent loop or by higher layer cells. b Equivalent block diagram representation: the P-S system is reduced to a first-order recurrent loop with time constant $\tau$, ignited by lateral geniculate nucleus (LGN) contributions $\left(e_{0}\right) . k_{\mathrm{a}}$ and $k_{\mathrm{d}}$ represent the spatial distributions of the pre- and postsynaptic sites, respectively, and $b$ is the inhibition strength 


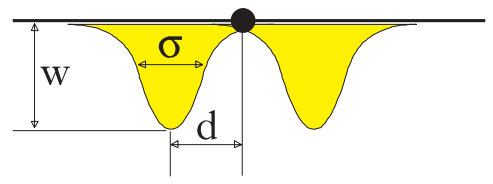

Fig. 2. The basic (symmetric) inhibitory kernel $k_{\mathrm{FB}}(x-\xi)$. The cell in the center receives inhibitory contributions from laterally distributed clusters of cells. The kernel is fully characterized by the values of three geometrical parameters $(\sigma, w, d)$ for each Gaussian function, associated with the spatial extent of the inhibitory couplings. The asymmetric kernels used in the model derive from this basic kernel by systematic variations of the geometrical parameters of one Gaussian function with respect to those of the other one

$$
\begin{array}{r}
k_{\mathrm{FB}}(x, y)=\frac{1}{2 \pi}\left(\frac{w_{1}}{\sigma_{1}^{2}} \exp \left[-\left(\left(x-d_{1}\right)^{2}+y^{2}\right) / 2 \sigma_{1}^{2}\right]\right. \\
\left.+\frac{w_{2}}{\sigma_{2}^{2}} \exp \left[-\left(\left(x+d_{2}\right)^{2}+y^{2}\right) / 2 \sigma_{2}^{2}\right]\right)
\end{array}
$$

This corresponds to a situation in which the selfinhibition of each neuron of the network is small compared with the mutual inhibition (i.e., reciprocal inhibition between different neurons of the network).

This work aimed to investigate how spatial asymmetries in the intracortical coupling function lead to nonseparable space-time interactions in the resulting discharge field of simple cells. To this end, we varied systematically the geometrical parameters $(\sigma, w, d)$ of the inhibitory kernel to consider three different types of inhibitory asymmetries: (1) different spatial spread of inhibition (i.e., $\sigma_{1} \neq \sigma_{2}$ ); (2) different amount of inhibition (i.e., $w_{1} \neq w_{2}$ ); (3) different spatial offset (i.e., $\left.d_{1} \neq d_{2}\right)$. A more rigorous treatment should also consider the continuous distortion of the topographic map (Mallot et al. 1990). In our analysis, this would result in a continuous deformation of the inhibitory kernel, but for the small distances over which inhibition occurs, the approximation of an uniform mapping is expected to produce only a negligible error.

\subsection{Parameter setting}

Architectural parameters were derived from the measured values of receptive fields of simple cells (Albus 1975; De Angelis et al. 1993a, 1995; Jones and Palmer 1987). Concerning the spatial domain, we fixed the size $\left(\sigma_{0}\right)$ of the initial receptive field (due to LGN contributions) for an 'average' cortical simple cell with a resultant discharge field of $\sim 5 \mathrm{deg}$; accordingly, we adjusted the parameters of the inhibitory kernel in order to account for spatial interactions only within the receptive field.

Considering the temporal domain, one should distinguish the time constant $\tau$, characterizing the population temporal response of intracortical interactions (P-S system), from the time constant $\tau_{0}$ characterizing the temporal response of 'individual' cells (i.e., closest to the temporal response of single cell membranes). In all the simulations, we set $\tau_{0}$ to $20 \mathrm{~ms}$, and we varied $\tau$ in the range $2-200 \mathrm{~ms}$.

\section{Results}

\subsection{The emergence of spatiotemporal receptive fields}

Due to the linearity assumption, the behavior of the network can be thoroughly analyzed by means of linear system theory. Accordingly, we can solve the system of integro-differential equations (1), (2), and (4) in the Fourier domain:

$E(\mathbf{u}, \omega)=H(\mathbf{u}, \omega) S(\mathbf{u}, \omega)$

with

$H(\mathbf{u}, \omega)=\frac{H_{0}(\mathbf{u}, \omega)}{1+j \omega \tau-b K_{\mathrm{FB}}(\mathbf{u})}=\frac{G_{0}(\omega) F_{0}(\mathbf{u})}{1+j \omega \tau-b K_{\mathrm{FB}}(\mathbf{u})}$

where $\mathbf{u}=(u, v)$ and $\omega$ represent the spatial and temporal frequency variables, respectively, and the upper-case letters refer to Fourier transforms. The transfer function $H(\mathbf{u}, \omega)$ represents the spectral response profile of the resultant receptive field $h(\mathbf{x}, t)$, interpreted as the spatiotemporal distribution of all the effects of cortical interactions. To avoid system instability, it is necessary that the interaction strength $b$ be kept below a threshold value $b_{\text {th }}$, thus preventing the denominator in (6) from becoming null.

For the sake of simplicity, in the following, we restrict the spatial analysis to a one-dimensional (1D) case, in the direction orthogonal to the preferred orientation of the receptive field. This $1 \mathrm{D}$ model produces spatiotemporal results $h(x, t)$ that are directly comparable with the spatiotemporal plots usually obtained by reverse-correlation studies (De Angelis et al. 1993a, 1995; McLean and Palmer 1989).

The spatial and temporal components of the resulting transfer function $H(u, \omega)$ are closely coupled considering that the spatial and temporal frequencies are no longer factorizable. The nature of this coupling strongly depends on the shape of the interaction kernel. Specifically, asymmetrical profiles of $k_{\mathrm{FB}}(x)$ give rise to complex forms of $K_{\mathrm{FB}}(u)=\operatorname{Re}\left\{K_{\mathrm{FB}}(u)\right\}+j \operatorname{Im}\left\{K_{\mathrm{FB}}(u)\right\}$ in (6) and hence to complex poles that result in spatiotemporal oscillatory behaviors. The presence of such poles is the determining factor of the generation mechanism of motion sensitivity. To aid discussion of this point, it is convenient to rewrite (6) as:

$H(u, \omega)=A(u) B(u, \omega) H_{0}(u, \omega)$

with

$\begin{array}{ll}A(u)=\frac{1}{1-b \operatorname{Re}\left\{K_{\mathrm{FB}}(u)\right\}} & \text { the spatial term and } \\ B(u, \omega)=\frac{1}{1+j \tau_{\mathrm{eff}}[\omega-\gamma(u)]} & \text { the spatiotemporal term }\end{array}$

where

$\tau_{\text {eff }}=\tau_{\text {eff }}(u)=\frac{\tau}{1-b \operatorname{Re}\left\{K_{\mathrm{FB}}(u)\right\}}$

is the effective time constant of the system resulting from the recurrence loop (see Fig. 3b), and 
a

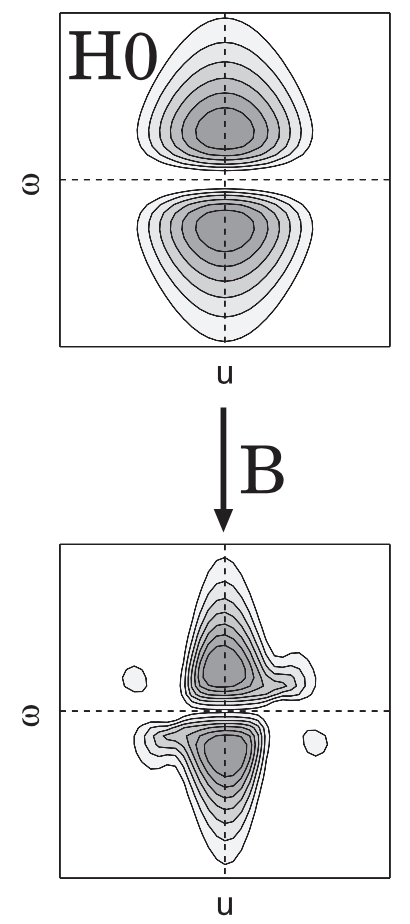

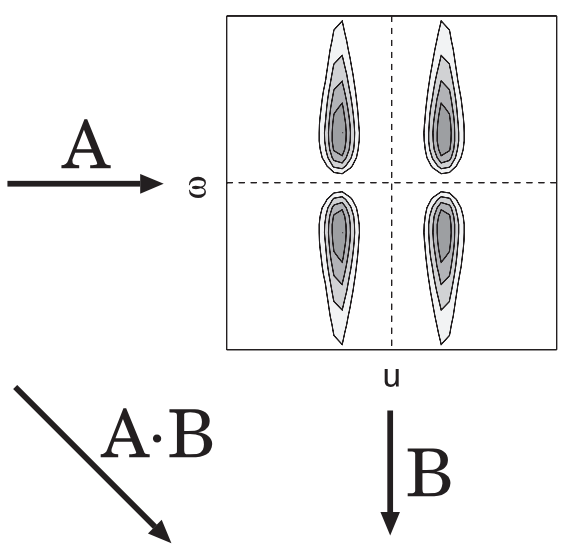

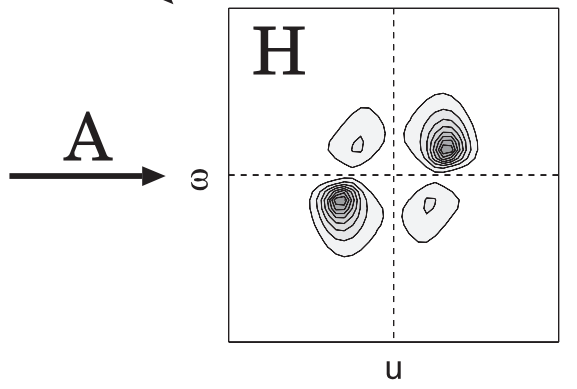

b

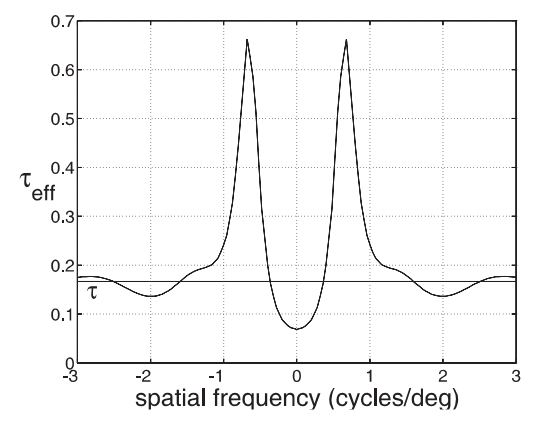

C

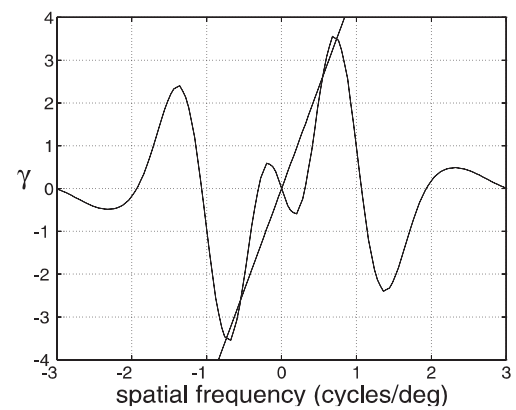

Fig. 3. a Schematic representation of the influence of recurrent inhibition on the spatiotemporal receptive field spectrum. The shearing of amplitude spectrum into two major blobs that lie along a diagonal in the odd quadrants derives from a redistribution of the spectrum energies of the LGN contribution $H_{0}$. The corresponding profiles of $\tau_{\text {eff }}$ and $\gamma$ as a function of the spatial frequency are evidenced in $\mathbf{b}$ and $\mathbf{c}$, respectively

$\gamma(u)=\frac{b \operatorname{Im}\left\{K_{\mathrm{FB}}\right\}}{\tau}$

is the value of the temporal frequency shift in the amplitude amplitude spectrum (see Fig. 3c). The real part of the recurrent kernel is primarily responsible for the low-pass to band-pass transition in the spatial domain, whereas the shift in the spectrum in the spatiotemporal frequency domain depends exclusively on its imaginary part. Specifically, considering the spatial term of the resulting operator $H(u, \omega)$, we observed that, for low values of the inhibition strength, the spectrum of $H(u, \omega)$ maintains a low-pass structure; for higher values of $b$, two peaks start growing approximately at $u^{*}= \pm 1 /\left(d_{1}+d_{2}\right)$, thus gaining a band-pass filter character that corresponds in space to an oscillatory receptive field profile closely resembling a Gabor function (Sabatini 1996). Concerning the effects of the spatiotemporal term in (7), if the dependence of $\gamma$ on the spatial frequency $u$ is approximately linear $(\gamma \simeq v u)$ (see Fig. 3c), at least in the range of frequencies where most of the spectrum energy is concentrated, the energy distribution along the spatial frequency $u$ shifts into the temporal frequency in $\omega^{*}=v u^{*}$, giving $H$ a sheared aspect corresponding to a specific direction selectivity. The system extracts energy from two blobs that lie along a diagonal through the origin; the spectral energy along this diagonal corresponds to the motion in a given direction and at the velocity $v=-\omega^{*} / u^{*}$. In Fig. $3 \mathrm{a}$, we show a pictorial description of how the spatial and spatiotemporal terms in (7) act on the initial operator $H_{0}(u, \omega)$ and mold its energy distribution in the resulting operator $H(u, \omega)$. It is worth noting that the working principle of recurrent inhibition differs from those of quadrature models (Adelson and Bergen 1985; Watson and Ahumada 1985), which are based on the summation of a pair of separable simple-cell receptive fields that are arranged in spatial and temporal quadrature. More specifically, the shearing of the energy spectrum into two major blobs that lie along a diagonal in the odd or the even quadrants derives from a redistribution of the spectrum energies, rather than implies a cancellation of parts of the spectrum, as occurs in quadrature models. Further general comments on the comparison of our approach to other models will be presented in Sect. 4.2.

\subsection{Structural asymmetries and direction tuning}

To better understand the role of the asymmetries and to discriminate among them, it is convenient to think of the recurrent inhibitory kernel $k_{\mathrm{FB}}$ as the sum of an even and an odd component:

$k_{\mathrm{FB}}(x)=k_{\text {even }}(x)+k_{\text {odd }}(x)$

where

$$
\begin{aligned}
& k_{\text {even }}(x)=\frac{k_{\mathrm{FB}}(x)+k_{\mathrm{FB}}(-x)}{2} \\
& \text { and } k_{\mathrm{odd}}(x)=\frac{k_{\mathrm{FB}}(x)-k_{\mathrm{FB}}(-x)}{2}
\end{aligned}
$$


The odd component is responsible for the imaginary part of $K_{\mathrm{FB}}$, whereas the even component is responsible for the real part of $K_{\mathrm{FB}}$.

The relative energy of the odd component, as compared with the total energy of the kernel, is indicative of the influence of the kernel's asymmetry on the slant of the spatiotemporal amplitude spectrum. More specifically, we can define a discriminative parameter:

$\Delta_{1}=\frac{\varepsilon_{\text {even }}-\varepsilon_{\text {odd }}}{\varepsilon_{\text {even }}+\varepsilon_{\text {odd }}}=\frac{\varepsilon_{\text {even }}-\varepsilon_{\text {odd }}}{\varepsilon_{\text {tot }}}=\frac{2 \int_{0}^{\infty} k_{\mathrm{FB}}(x) k_{\mathrm{FB}}(-x) d x}{\int_{0}^{\infty}\left[k_{\mathrm{FB}}^{2}(x)+k_{\mathrm{FB}}^{2}(-x)\right] d x}$

that varies in the range from 0 (i.e., fully asymmetric kernel) to 1 (i.e., symmetric kernel).

If we recall that the shift value $\gamma(u)$ is most effective when high shift values are in the range of frequencies where the spectrum energy peaks, we can evaluate the efficiency of translation by introducing a second discriminative parameter, $\Delta_{2}$, related to the balance of the right-hand and left-hand parts of the connection kernel:

$\Delta_{2}=\frac{\varepsilon_{+}-\varepsilon_{-}}{\varepsilon_{+}+\varepsilon_{-}}=\frac{\varepsilon_{+}-\varepsilon_{-}}{\varepsilon_{\mathrm{tot}}}=\frac{4\left|\int_{0}^{\infty} k_{\mathrm{even}}(x) k_{\mathrm{odd}}(x) d x\right|}{\int_{0}^{\infty}\left[k_{\mathrm{FB}}^{2}(x)+k_{\mathrm{FB}}^{2}(-x)\right] d x}$

where $\varepsilon_{+}$and $\varepsilon_{-}$are the right-hand and left-hand energies, respectively. $\Delta_{2}$ ranges from 0 (i.e., balanced kernel) to 1 (i.e., fully unbalanced kernel) and is related to the degree of similarity (i.e., correlation) between the odd and even components of the coupling kernel. A high degree of similarity implies that the the real and imaginary parts of the energy spectrum of $k_{\mathrm{FB}}$ will peak in the same range of frequencies.

In order to analyze comparatively the tuning properties of the modeled cortical cells for different values of the parameters $\Delta_{1}$ and $\Delta_{2}$, we define a direction index (DI) as a quantitative measure of the directionally selective response to drifting sine-wave gratings. The DI is defined as the difference between the response amplitudes in the preferred (P) and non-preferred (NP) directions, normalized by $\mathrm{P}$ :

$\mathrm{DI}=\frac{\mathrm{P}-\mathrm{NP}}{\mathrm{P}}$

Given the linearity of our model, DI can be straightforwardly derived from the amplitude spectra of the resulting receptive fields. $\mathrm{P}$ is evaluated as the peak value of the spectrum and corresponds to the amplitude response of a cell to a drifting sine-wave grating characterized by the wave vector $\left(u^{*}, \omega^{*}\right)$; NP corresponds to the value of the amplitude spectrum in $\left(u^{*},-\omega^{*}\right)$. By convention, DI is negative if the preferred direction has a leftward component. A more sound measure for direction selectivity that takes into account the variability of the DI according to the velocity of the stimulus is the mean direction index (MDI), defined as the weighted average of the direction indices over a significant range of velocities, and the responses $\left(\mathrm{P}_{i}\right)$ in the preferred direction are used as weighting factors (Orban 1984):
$\mathrm{MDI}=\frac{\sum_{i=1}^{N} \mathrm{P}_{i} \mathrm{DI}_{i}}{\sum_{i=1}^{N} \mathrm{P}_{i}}$

where $\mathrm{DI}_{i}$ is the direction index at the $i$ th velocity, and $N$ is the number of velocities for which the direction index has been computed. The contour plot in Fig. 4 illustrates the dependence of the MDI on the type and degree of kernel asymmetries, as summarized by the parameters $\Delta_{1}$ and $\Delta_{2}$. The remaining parameters of the model are set to typical values (e.g., the inhibition strength is set to $75 \%$ of its specific threshold value); their influence on the MDI is discussed in the next section. The most influential parameter is $\Delta_{1}$ : the highest values of the MDI are obtained for low values of $\Delta_{1}$, whereas the MDI is almost unaffected by the balance parameter $\Delta_{2}$. These facts point to the importance of asymmetries for the emergence of direction selectivity.

In general, the inhibitory kernel corresponding to a specific couple of values $\left(\Delta_{1}, \Delta_{2}\right)$ is characterized by a particular mixture of asymmetries in all its geometrical parameters $(\sigma, d, w)$. To distinguish the different role of the three types of asymmetries, we superimpose the loci corresponding to variations in single geometrical parameters (dashed lines in Fig. 4). In particular, we chose four couples of $\left(\Delta_{1}, \Delta_{2}\right)$ (indicated by asterisks in Fig. 4) as representative of different kinds of asymmetries.

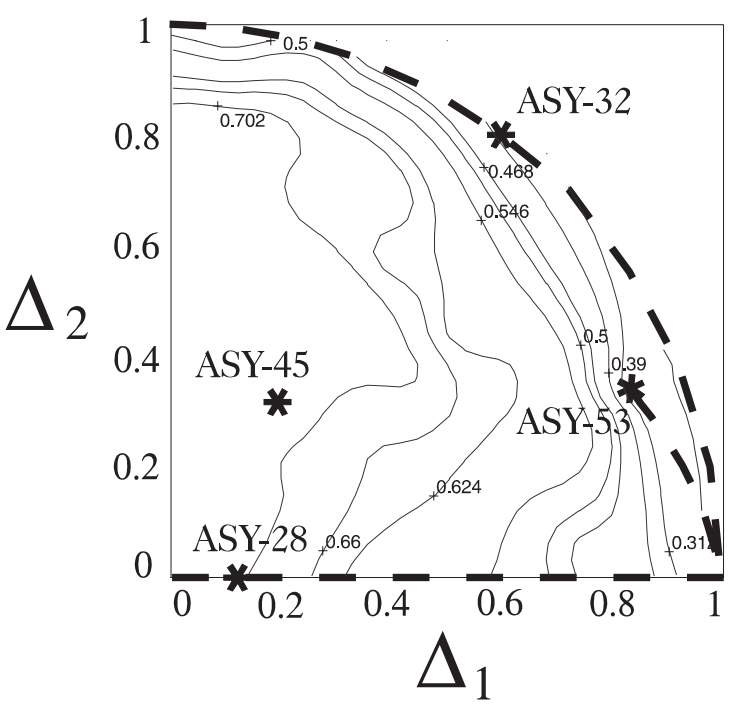

Fig. 4. Contour plot of the mean direction index (MDI) on the $\left(\Delta_{1}, \Delta_{2}\right)$ plane, when the inhibition strengths $b$ are set to $75 \%$ of their threshold values, $\tau_{0}=20 \mathrm{~ms}$, and $\tau=10 \mathrm{~ms}$. Each couple of values $\left(\Delta_{1}, \Delta_{2}\right)$ is related to a specific asymmetry of the inhibitory kernel, obtained by choosing the architectural parameters $(\sigma, d, w)$ of one Gaussian different from those of the other. Asterisks indicate couples of $\left(\Delta_{1}, \Delta_{2}\right)$ representative of different asymmetries. Bold dashed lines represent the loci of points on the $\left(\Delta_{1}, \Delta_{2}\right)$ plane corresponding to variations in a single geometrical parameter of the inhibitory kernel: The horizontal line coincident with the $\Delta_{1}$ axis corresponds to variations in the spatial offset $\left(d_{1} \neq d_{2}\right)$; the small arc departing from $(1,0)$ corresponds to variations in the spatial spread $\left(\sigma_{1} \neq \sigma_{2}\right)$; the long arc from $(1,0)$ to $(0,1)$, which bounds the MDI surface, corresponds to variations in the amount of inhibition $\left(w_{1} \neq w_{2}\right)$ 


\section{type of asymmetry}
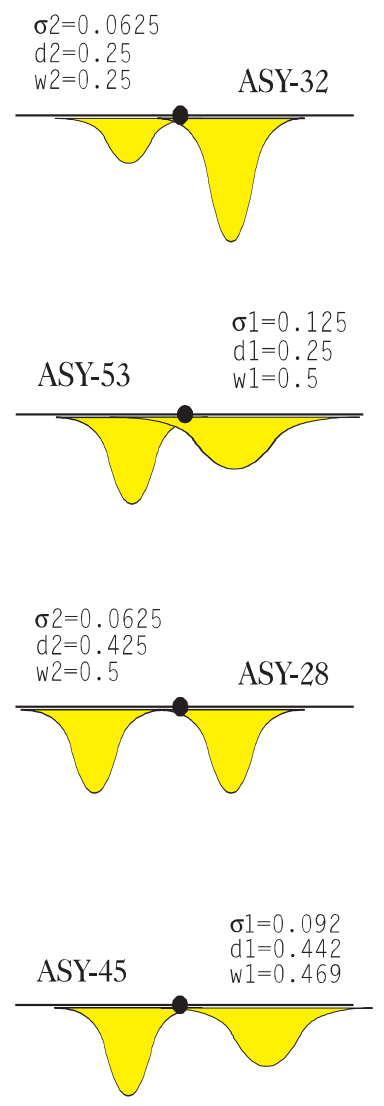

amplitude spectrum
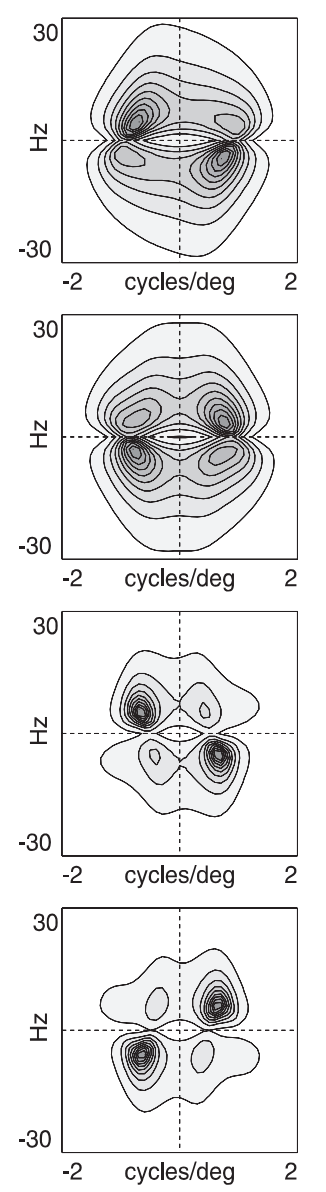
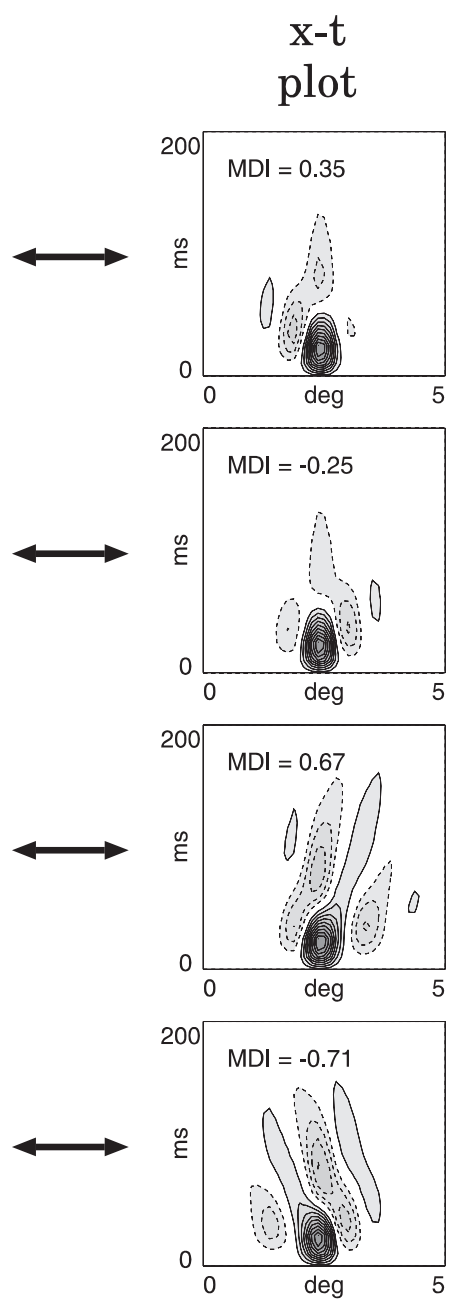

Fig. 5. Typical receptive fields for two nondirectional cells (ASY-32 and ASY-53) and two direction-selective cells (ASY-28 and ASY-45), obtained from the corresponding inhibitory coupling kernels depicted on the left. The $x$-t plots in the right column are obtained by numerical Fourier transform of the amplitude spectra. Solid lines indicate excitatory subregions, whereas dashed lines indicate inhibitory sub-regions. The time constants used are $\tau_{0}=$ $\tau=20 \mathrm{~ms}$. The inhibition strength $b$ is $75 \%$ of its threshold value $b_{\text {th }}$
In Fig. 5, we show the four asymmetric intracortical kernels considered, together with the corresponding receptive fields resulting from our model. As previously reported (Sabatini et al. 1996), asymmetries in the spatial offset of the cortical inhibitory sites (e.g., ASY-28 and ASY-45) are more effective in inducing a high direction selectivity than other types of asymmetries (e.g., ASY -32 and ASY-53), as is evident from the resulting spectra characterized by a marked motion-type nonseparability. For a more intuitive understanding of how the model cells respond to stimuli in motion, we carried out the inverse Fourier transform of the spatiotemporal spectra. This yields the spatiotemporal weighting functions or spatiotemporal receptive fields shown in the right column of Fig. 5. The shape of the spatiotemporal receptive fields of direction-selective cells (e.g., ASY-28 and ASY-45) is basically similar. They are elongated and oriented in space-time at an orientation which corresponds to the drift velocity of the test grating. Each field is made up of several subregions of alternating polarity, produced by the modulation in space and time. The receptive field of a non-direction-selective cell (e.g., ASY-32 and ASY-53) is quite different from the others. It is not oriented in space-time and has a preferred velocity proximal to 0 $\mathrm{deg} / \mathrm{s}$. Along the spatial axis, it has subregions of alternating polarity, but along the temporal axis it does not.

\subsection{Parametric variations and response diversity}

Up to now, we have investigated the effects on direction selectivity of different choices of kernel parameters $(\sigma, d, w)$ that correspond to specific patterns of intracortical couplings, having set the other parameters of the model to typical values. Although the specific shape of the inhibitory kernel determines the qualitative behavior of the system, we should evaluate the efficacy of the recurrent scheme on direction selectivity also in relation to the values of the inhibitory strength and of the loop dynamics. Accordingly, we varied the percentage of inhibition strength $b$, as compared with its threshold value $b_{\text {th }}$, and the time constant of the recurrent loop $\tau$ related to the time constant of LGN contributions $\tau_{0}$. The spatiotemporal plots of the resulting receptive fields $h(x, t)$ are tabulated in Fig. 6. According to the classification proposed by Orban (1984), we distinguish three classes of visual cortical cells: nondirectional (MDI <0.5), directional asymmetric $(0.5 \leq$ MDI $<0.66$ ), and direction-selective (MDI $\geq 0.66$ ). Increments of $b$ enhance the effects of inhibition and result in larger MDIs. Moreover, we observe that for motionsensitive cells (e.g., ASY-28 and ASY-45), strong direction selectivity can be obtained when the values of the $\tau / \tau_{0}$ ratio are in the range $0.5-2.0$ (e.g., $\tau=10-40 \mathrm{~ms}$, and 
ASY-32

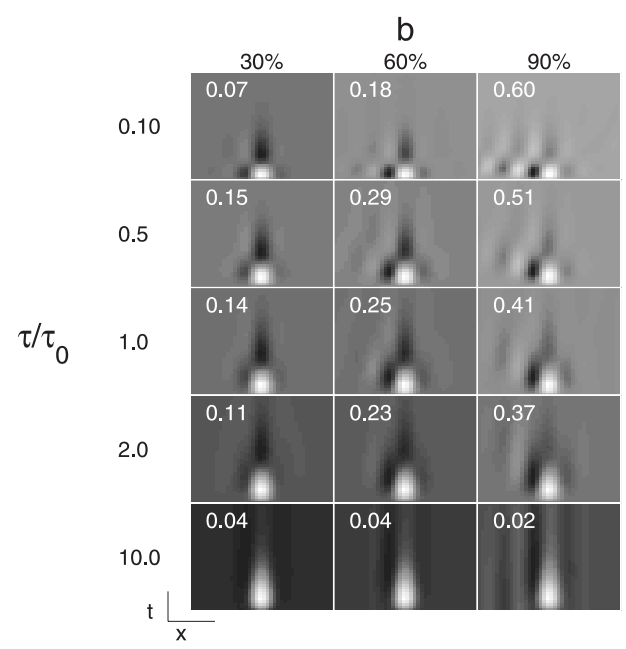

ASY-28

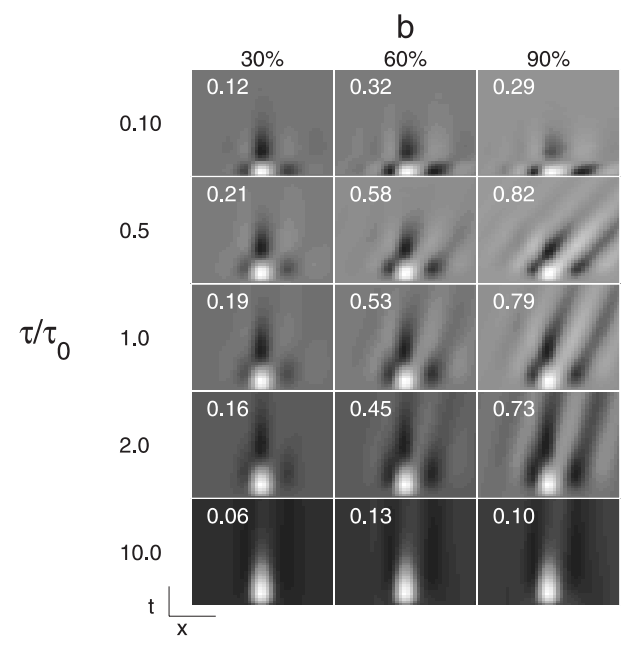

ASY-53

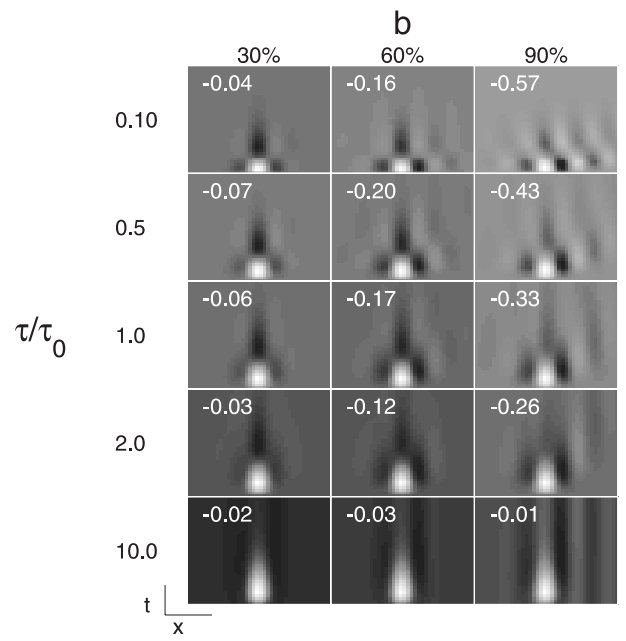

ASY-45

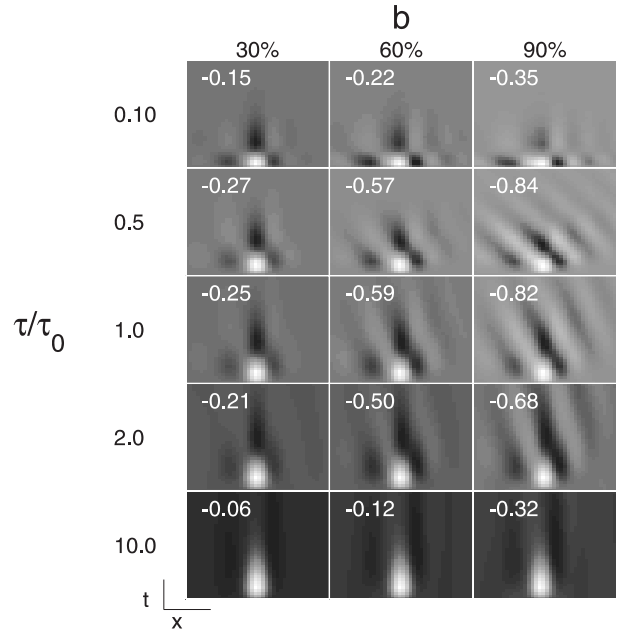

Fig. 6. Spatiotemporal receptive fields resulting from systematic variations of the percentage of inhibition strength $b$, as compared with its threshold value $b_{\mathrm{th}}$, and of the time constant of the recurrent loop $(\tau)$ with respect to the geniculate time constant $\left(\tau_{0}\right)$, for the four asymmetries considered. The MDI as defined by (14) is shown for each spatiotemporal plot. Note that by convention the MDI is negative if the preferred direction has a leftward component $\tau_{0}=20 \mathrm{~ms}$ ); with larger or lower values of the $\tau / \tau_{0}$ ratio, such cells lose their direction selectivity. On the contrary, nondirection-selective cells (e.g., ASY-32 and ASY-53) achieve only a poor direction-asymmetric behavior for very low values of the $\tau / \tau_{0}$ ratio. The corresponding velocity response curves are tabulated in Fig. 7. According to Orban's classification, the different shadings of the boxes indicate different degrees of direction selectivity (darkest gray corresponds to nondirection-selective cells). A strong direction selectivity (MDI $\geq 0.66$ ) is usually associated with velocity-tuned cells. Though other classes of cells are also present, these are predominantly nondirection-selective or directional asymmetric (Orban 1984; Wörgötter and Holt 1991). It is worth noting that increasing the $\tau / \tau_{0}$ ratio strengthens the velocity low-pass behavior, even when the MDI values are still quite high. Moreover, by decreasing the inhibition strength $b$, the velocityresponse curve broadens. The range of optimal velocities we can obtain spans from $0 \mathrm{deg} / \mathrm{s}$ up to more than $100 \mathrm{deg} / \mathrm{s}$, depending on the time constants used and on the size of the initial receptive field. Increments of the value of the time constants of the model lower the optimal velocity, whereas the opposite occurs for increments of the size of the initial receptive field (cf. Wörgötter and Holt 1991).

To validate the values of the direction selectivity indices we obtained with our model, let us briefly discuss the influence of the model's parameters on the activation levels of our cells. Concerning orientation selectivity, David Somers (Somers et al. 1995) reported that the activity of his cells would go basically to zero when using only inhibition to obtain realistic orientation tuning. The large MDIs we observe for high values of inhibition strength $b$ are instead still associated with a significant degree of activation. This peculiarity of our model derives from the particular spatial distribution of recurrent inhibitory interactions. Indeed, the small percentage of self-inhibition compared with the inhibition received by neighboring cells (let us recall that the inhibitory coupling kernel $k_{\mathrm{FB}}$ peaks at a certain distance from its center) prevents the activation level from being reduced by the inhibition. On the contrary, high values of inhibition strength $\left(b>b_{\text {th }}\right)$ will lead to unstable behavior, 
ASY-32

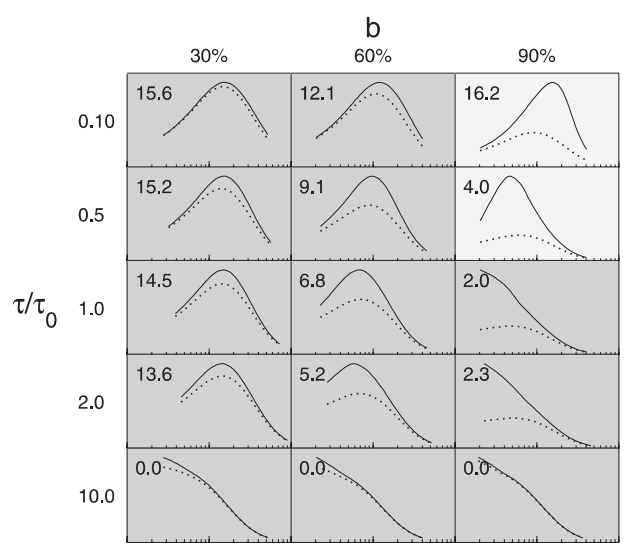

ASY-28

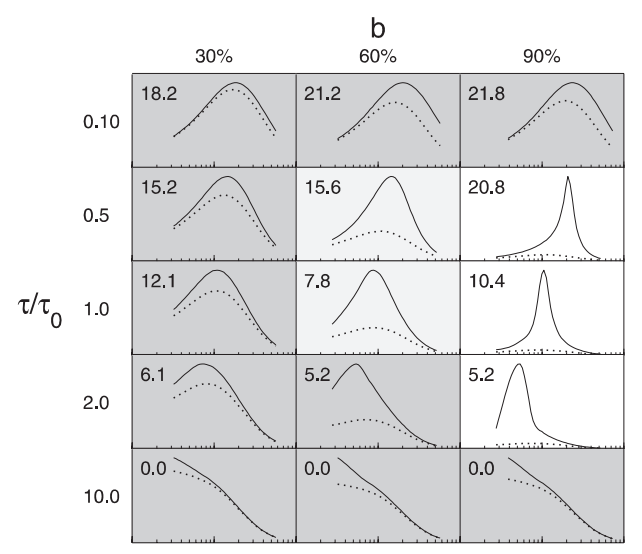

ASY-53

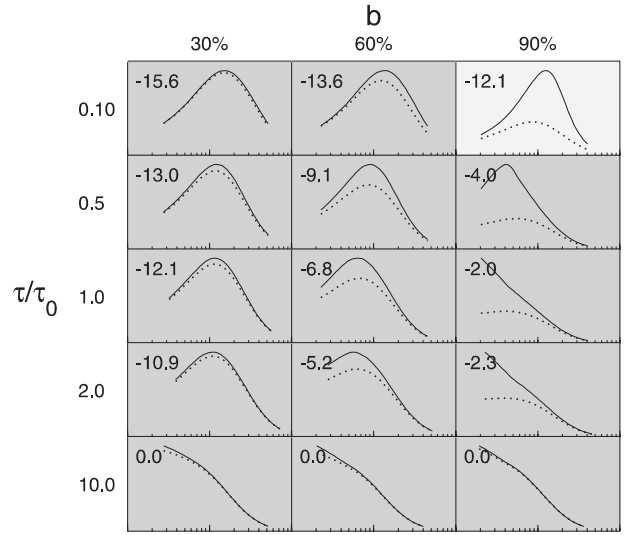

ASY-45

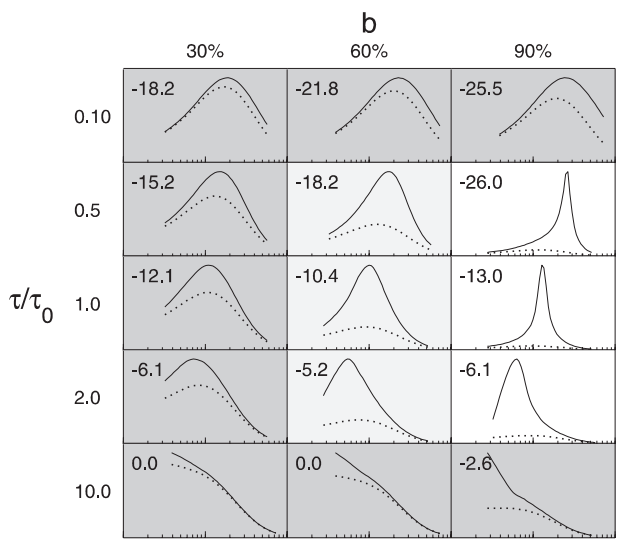

Fig. 7. Effects on the velocityresponse curves of the systematic variations of the model's parameters $\tau$ and $b$, as in Fig. 6. Curves represent the normalized cell responses to drifting gratings with different velocities, marked with a logarithmic scale from 1 to 100 cycles/deg. For each curve, the optimal velocity value is indicated. By convention, rightward direction of motion corresponds to positive velocities. Solid lines represent the responses in the preferred direction, while broken lines represent the responses in the nonpreferred directions. Different shadings of the boxes emphasize different types of direction selectivity (lighter gray corresponds to higher direction tuning) thus resulting in an increased amplitude level and in an increased width of the receptive field. The activation level is de facto insensitive to variations of the inhibition strength $b$, when it is kept below its threshold value $\left(b<b_{\text {th }}\right)$.

The situation is different for variations of cell dynamics. In this case, we observe decreasing amplitude signals as the time constant of the recurrent loop $\tau$ increases with respect to that of the geniculate input $\tau_{0}$. The decrease of the activation value is associated with the temporal low-pass characteristics of the neural layer (a leaky integrator with time constant $\tau$ ). This behavior can be understood intuitively by observing that for the recurrent inhibition to be effective, sufficient time is required for it to be fed back. For fast-changing geniculate inputs $\left(\tau / \tau_{0} \gg 1\right)$, the inhibition may not keep up, and thus resulting activation patterns are significantly attenuated. However, this does not represent a true problem since real cortical cell dynamics cannot vary over so broad a range of values, which has been here considered only to characterize the behavioral trends of the network. It is worth noting that in Fig. 6, to best represent the spatiotemporal organization of the resulting receptive field profiles for a $\tau / \tau_{0}$ ratio that varies over two orders of magnitude, we decided to represent them by using, for each plot, the whole range of gray levels.

\subsection{Conjoint spatiotemporal system behavior}

In Sect. 3.1, we observed the rising of complex poles due to the imaginary component of the Fourier transform of the recurrent interaction kernel $K_{\mathrm{FB}}(u)$ in the denominator of the transfer function $H(u, \omega)$. The presence of these poles turns out to be the determining factor in the formation of the specific spatiotemporal coupling which underlies motion sensitivity. As it appears from the relationships obtained for $\tau_{\text {eff }}(u)$ and $\gamma(u)$, (8) and (9), the system shows different temporal behaviors when excited by gratings with different spatial frequencies. To investigate quantitatively the nature of this spatiotemporal coupling and how it influences the response properties of the resulting receptive fields, we can write, in the Laplace domain $(s=v+j \omega)$, the temporal response of the system for a set of $N$ spatial frequencies (i.e., complex phasors), uniformly distributed over the range in which we are interested in characterizing the system. By considering both positive and negative spatial frequencies (i.e., pairs of conjugate phasors), for each frequency $\pm u_{i}$ the system responds with a pair of complex conjugate poles $p_{i}$ and $p_{i}^{*}$. Therefore, the resulting filter can be expressed as a sum of (decoupled) second-order systems, each of which determines the temporal evolution of the related spatial frequency component of the output: 
$\tilde{H}(s)=G_{0}(s) \cdot \sum_{i=1}^{N} F_{0}\left(u_{i}\right) \frac{\left(s-z_{i}\right)}{\left(s-p_{i}\right)\left(s-p_{i}^{*}\right)}$

where

$z_{i}=-2 / \tau_{\mathrm{eff}}\left(u_{i}\right)$

$p_{i}=-1 / \tau_{\mathrm{eff}}\left(u_{i}\right)-j \gamma\left(u_{i}\right) ; \quad p_{i}^{*}=-1 / \tau_{\mathrm{eff}}\left(u_{i}\right)+j \gamma\left(u_{i}\right)$

In order to compare the contribution of each term to the sum, it is convenient to introduce the normalized system response

$\tilde{H}_{i}(s)=\frac{1}{\sqrt{E_{i}}} \frac{\left(s-z_{i}\right)}{\left(s-p_{i}\right)\left(s-p_{i}^{*}\right)}$

where $E_{i}$ is the energy of the second-order system at spatial frequency $u_{i}$. Therefore, (16) becomes:

$\tilde{H}(s)=G_{0}(s) \cdot \sum_{i=1}^{N} c_{i} \tilde{H}_{i}(s)$

where $c_{i}=F_{0}\left(u_{i}\right) \sqrt{E_{i}}$.

The $c_{i}$ coefficient weights the importance of the contribution of the spatial eigenmode, $u_{i}$, to the network response. For spatial frequencies below the cut-off frequency of $F_{0}$, the highest values of $c_{i}$ are associated with the strongest temporal responses, which we call resonant modes of the system or resonant peaks. The presence of resonant peaks around a specific temporal frequency, in correspondence with a restricted range of (pass band) spatial frequencies, implies a specific tuning in the spatiotemporal frequency domain. However, the efficacy of such a configuration will depend also on the position of the corresponding complex conjugate poles with respect to the imaginary axis and the open loop poles associated with the LGN contribution $G_{0}(s)$. To this end, we consider the closed loop contour pole diagrams representing the positions in the $s$-plane of the complex poles $p_{i}$ and $p_{i}^{*}$ as a function of the spatial frequencies $u_{i}$. In Fig. 8, we show two typical contour pole diagrams for a nondirectional and a direction selective cell. For the sake of clarity, only the parts of the diagrams corresponding to positive spatial frequencies are reproduced, whereas the specular parts, corresponding to negative spatial frequencies, are represented by dotted lines. The shading used to fill the squares codes for the value of $c_{i}$ associated with each pole: the darkest grays correspond to resonant modes. The system is stable if and only if all of the poles of the spatiotemporal transfer function lie in the open left-hand side of the complex plane. The dynamic behavior of the system is mostly determined by complex conjugate poles close to the imaginary axis (dominant poles). Indeed, dynamic behaviors associated with other poles farther from the imaginary axis are of minor importance, and their contributions decay more rapidly.

By examining the contour pole diagrams in Fig. 8, we can infer some architectural design principles underlying cortical cell motion sensitivity and interpret the receptive fields reported in Sect. 3. For nondirec-
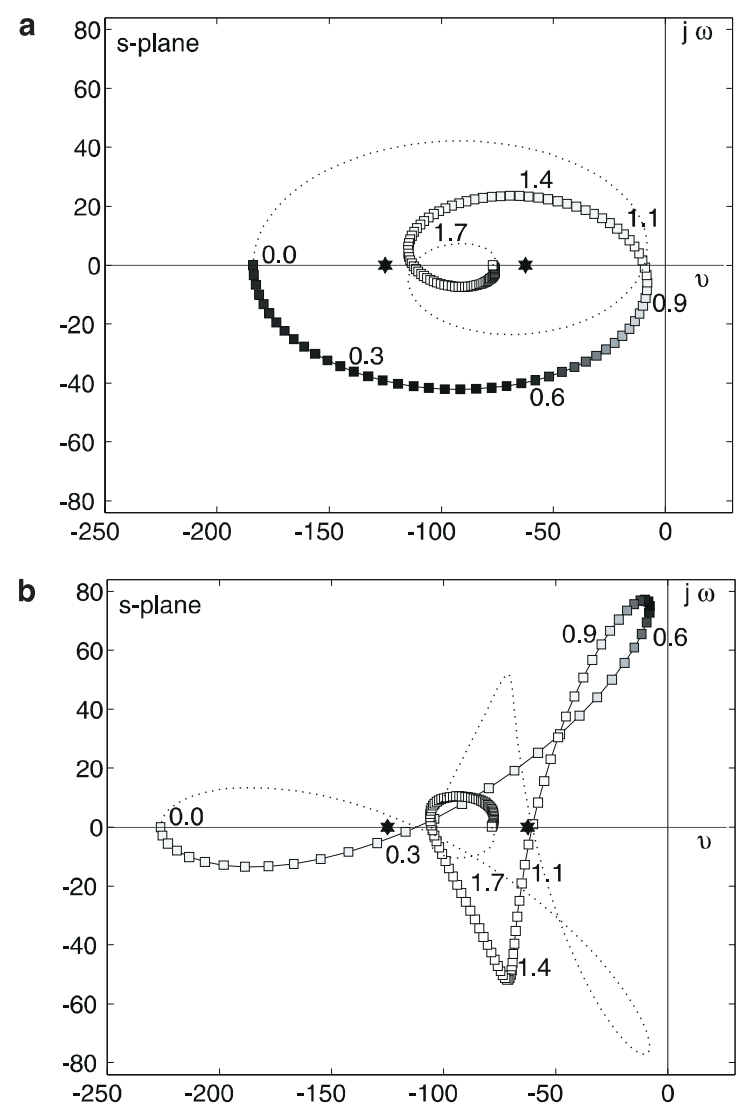

Fig. 8. Contour pole diagrams of the closed loop complex poles $p_{i}$ of the network as a function of positive spatial frequencies (a) for a nondirectional cell (ASY-32) and (b) for a direction-selective cell (ASY-45). The importance of each pole with respect to the spatial response modes of the system is coded by the gray level of the shading used to fill the squares $(\square)$ (the darker the shading, the greater the importance of the corresponding pole). The diagrams were obtained by uniform spacing of the spatial frequency that was considered as a parameter and varied from 0 to 3 cycles/deg. The curves are graduated by values of the spatial frequency. The specular parts of the diagrams corresponding to the variations of the conjugate poles $p_{i}^{*}$ as a function of negative spatial frequencies are represented as dotted lines. Asterisks $(*)$ represent the open loop double poles of the system

tional cells, the poles corresponding to resonant modes span a broad range of temporal frequencies, thus indicating that the system is not tuned to any specific temporal mode but has different temporal responses depending on the spatial frequency component of the input signal. Moreover, their effect is masked by the open loop poles which are closer to the imaginary axis. A different pattern is obtained for direction-selective cells: The contour diagram twists into a branched structure, and complex poles concentrate within a restricted area of the $s$-plane, proximal to the imaginary axis. This yields dominant temporal modes characterized by a limited set of temporal frequencies. This behavior, for direction-selective cells, reflects the low-pass to band-pass transition both in space and time caused by the inhibitory feedback that gives the skewed aspect to the amplitude spectrum that characterizes motion sensitivity (cf. Fig. 3a). 
a

TRANSIENT

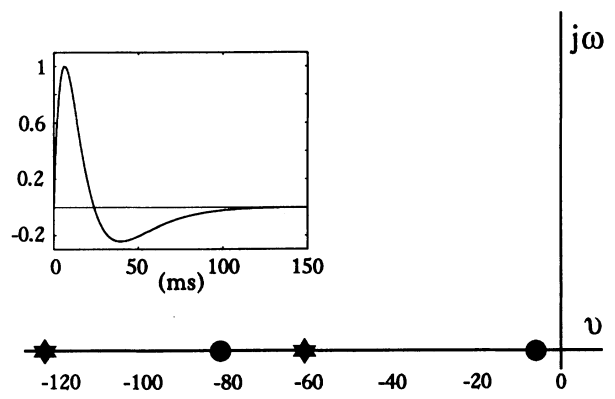

b
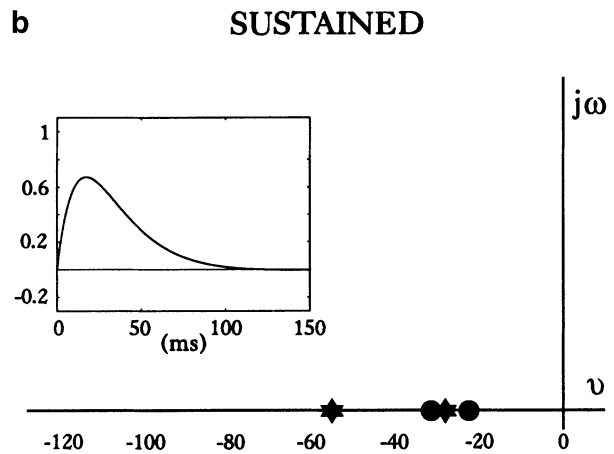

\subsection{Direct influences of geniculate inputs}

The contour pole diagrams (derived in the previous section) represent intrinsic characteristics of the cortical network by giving the response components associated with the feedback loop only. In direction-selective cases, although such components are usually dominant, their amplitudes can be influenced by the pattern of the fixed (i.e., invariant with spatial frequency $u_{i}$ ) open loop poles and zeros due to the LGN contributions. Hence, different choices of the LGN temporal responses may produce different behaviors of the overall system $\tilde{H}(s)$. A complete investigation of the effects of different LGN inputs is beyond the scope of this work. However, an interesting case study concerns the analysis of the final value response of the model simple cells in relation to transient and sustained types of LGN inputs. The choice of the parameters in (3) adopted so far $\left(\alpha_{1}=8 \mathrm{~ms}\right.$, $\left.\alpha_{2}=16 \mathrm{~ms}, L=0.9\right)$ characterizes the time response of a transient-type LGN cell. With a different parameter setting $\left(\alpha_{1}=16 \mathrm{~ms}, \alpha_{2}=32 \mathrm{~ms}, L=0.1\right)$, we obtain a time response that approximates that of a sustained-type LGN cell. In Fig. 9, we show the temporal behaviors of the geniculate inputs together with the velocity-response curves of the model simple cells driven by the corresponding inputs, for different asymmetries. The responses for sustained-input cells (see Fig. 9b) show a shift of the velocity-response curves to lower speeds, leading to velocity low-pass behavior that is not observed (see Fig. 9a) for transient-input cells (cf. Orban 1984, Fig. 8/5).

\footnotetext{
${ }^{1}$ The influence of the closed loop zeros is ignored.
}

Fig. 9a,b. Direct influences of the LGN inputs on the velocity-response curves of simple cells. On the right, the velocity-response curves of simple cells obtained for different types of asymmetries, when driven by transient (a) and sustained (b) LGN inputs. The different temporal characteristics of LGN contributions in time and Laplace domains $(s=$ $v+j \omega)$ are shown on the left: open loop poles are represented as asterisks (*), and open loop zeros are shown as solid circles $(\bullet)$. Transient-type LGN inputs facilitate the formation of velocity-tuned simple cells, whereas sustained-type LGN inputs facilitate the formation of lowpass velocity simple cells

\subsection{Effects of additional recurrent excitation}

Up to now, we have analyzed the effects of purely inhibitory intracortical interactions. However, local cortical circuits include dense recurrent connections among spiny stellate cells (Douglas et al. 1989, 1995; Stratford et al. 1996). Such connections provide a significant source of recurrent excitation and have been suggested to be very effective in generating cortical direction selectivity (Suarez et al. 1995). Therefore, we incorporated additional excitatory couplings (modeled by an extra positive Gaussian function) into the recurrent kernel $k_{\mathrm{FB}}$, and we varied systematically both its displacement $(d)$ related to the center and its weight $(w)$. The resulting shape of the interaction kernel resembles a local connectivity scheme based on shortrange excitatory and longer-range inhibitory connections that have been extensively used in the neural network literature (Amari 1977; Grossberg 1976; von der Malsburg 1973; Xing and Gerstein 1996). The results (not shown here) confirmed the importance of recurrent excitation for the refining of direction selectivity, provided that excitation is spatially displaced from the location of the target cell. Specifically, simple cells show higher MDIs and are tuned to higher velocities when excitation originates from the side where the inhibitory couplings are the weakest (i.e., the faintest or the farthest); on the contrary, simple cells lose direction selectivity so much that they show a reversal in the preferred direction when excitation arises from the opposite side. Symmetrically centered excitation always results in a considerable worsening of direction selectivity. The results agree with recent models of cortical 
direction selectivity based on massive recurrent excitatory interactions (Suarez et al. 1995), and they also substantiate the importance of asymmetric interactions for visual cortical direction tuning (cf. also von Seelen et al. 1987).

\section{Discussion}

Our architectural model can be characterized as a linear model of homogeneous layers with feedback. The linearity assumption, together with the model's essentiality, allows a detailed analysis of the basic mechanism of directionality in the Fourier domain. The analysis has shown that spatial asymmetries in the intracortical interactions are sufficient to generate direction selectivity without any additional feedforward direction selectivity bias. By varying the model's parameters, we obtained different types of direction selectivities and velocity responses that closely match experimental data.

\subsection{Interpretation of the resultant receptive field}

When stimulated with an external signal $s(\mathbf{x}, t)$, the network reacts with a distribution of activity $e(\mathbf{x}, t)$ that corresponds to the neural representation of the stimulus (Mallot 1995). Specifically, the spatiotemporal point image (i.e., impulse response) obtained by inverse Fourier transform of the network transfer function (6) represents the distribution of activation of the layered network when externally stimulated by a Dirac function $\delta(\mathbf{x}, t)$ in space and time (e.g., a small spot of light briefly flashed on the retina). Since the model is linear and space-invariant, the impulse response may be identified with the weighting function of the receptive field of any unit of the neural layer. One drawback of this approach is that the population of model neurons is unrealistically homogeneous. Thus, the model cannot represent the individual behavior of single cortical cells, but rather indicates basic general properties probably common to all or most neurons within the averaging area. The overall spatiotemporal filtering operation performed by the continuous network can be compared with the spatiotemporal flow of excitation revealed by current source density analysis (Luhmann et al. 1990a,b; Nicholson 1973), local field potentials (Eckhorn et al. 1993; Lohmann et al. 1988), or optical imaging (Arieli et al. 1995; Grinvald et al. 1994).

We explored the information processing capabilities of these spatiotemporal patterns of activity in relation to the construction of spatiotemporal receptive fields in the primary visual cortex. The positive and negative subregions of the resultant average receptive field correspond to excitatory and inhibitory influences on the cell's response, respectively. To be consistent with the spatiotemporal profiles measured through responses to complementary stimuli (e.g., bright and dark stimuli), one assumes that inhibition elicited by a bright stimulus is equivalent to excitation elicited by a dark stimulation, and vice versa. Accordingly, the inhibitory subregions could also be interpreted as dark subregions (i.e., regions which are responsive to the onset of a dark stimulus). This is a common measurement assumption (De Angelis et al. 1993a, Jones and Palmer 1987) that yields linear estimations of receptive field profiles that can be directly compared with our results.

\subsection{Comparison to other models}

Various models have been proposed to describe the origin of direction selectivity in the primary visual cortex and, more generally, to seek to answer the question of how cortical simple cells respond to motion signals. This issue has been considered from two distinct perspectives.

From a computational perspective, the problem of motion detection can be redefined to the problem of measuring local specific spatiotemporal correlation in the input signal. Within a linear framework, this corresponds to detecting, tilted edges in space-time dimensions by spatiotemporally oriented linear filters (Adelson and Bergen 1991). Hence, filters with appropriately oriented impulse responses (or cells with appropriately oriented receptive fields) will selectively respond to motion in particular directions. Pioneering work in this field has been conducted by Adelson and Bergen (1985) and by Watson and Ahumada (1985) (see also Wilson 1985), who also suggested simple feedforward neural mechanisms to construct directionally tuned receptive fields, by adding together the outputs of two separable filters in quadrature both in spatial and temporal domains. Over the years, these 'quadrature models' have been improved to account for more accurate aspects of simple cell responses to visual motion, by alternating linear filtering operations with point nonlinearities such as rectifications, squaring operations, threshold-like nonlinearities, normalization stages, etc. (Carandini and Heeger 1994; Emerson 1997; Emerson and Huang 1997; Heeger 1993, Wilson 1985). All computational models, both linear and linear-nonlinear, follow a neuromorphic filter design approach that behaviorally characterizes simple cells at the level of their receptive fields, but disregards anatomical and electrophysiological facts on the basis of their response properties.

On the contrary, from an architectural point of view, one tends to emphasize the detailed circuitry and the dynamics of cortical networks. Realistic biophysical models of a part of the primary visual pathway, with tens of thousands of integrate-and-fire neurons connected in networks, have been recently proposed and their function investigated through extensive computer simulations (Maex and Orban 1996; Somers et al. 1995; Suarez et al. 1995; Wehmeier et al. 1989; Wörgötter and Koch 1991). Orientation specificity and direction selectivity of simple cells have been revealed as emergent properties of massive excitatory and inhibitory interactions [cf. canonical cortical microcircuit (Douglas et al. 1989)]. Due to the computational complexity (high-dimension) of the system of coupled nonlinear partial differential equations at stake, analytical predictions of 
the network's behavior have only rarely been attempted, under heavy simplifying assumptions, and always restricted to basic functioning mechanisms of cortical microcircuits (Douglas et al. 1995; Maex and Orban 1992, 1996; Suarez et al. 1995).

The model we present in this paper is based on architectural considerations, but adopting a linear mean field description, it can be straighforwardly reduced to a computational model that is directly comparable (see Sect. 3.1) with those of Adelson and Bergen (1985) and of Watson and Ahumada (1985). More specifically, we changed from an architectural description of cortical function, in which a simple cell's response was a combination of geniculate and intracortical contributions, to a behavioral description in which we relate the simple cell response directly to the stimulus intensity, through an equivalent feedforward linear operator. Therefore, our model combines the simplicity of feedforward linear filtering approaches with a certain degree of structural specificity of cortical networks, by incorporating more advanced architectural principles, such as lateral interactions and recurrent (i.e., feedback) computation (cf. Mallot and Giannakopoulos 1996; von Seelen et al. 1987).

As for quadrature models, additional nonlinearities in cascade could, of course, improve the functionality of our model, too, but a detailed analysis on this point is beyond the scope of this paper.

\subsection{Conclusions}

The field approach allows a concise mathematical treatment of the basic architectural principles incorporated in the model. Though very simplistic, the model represents a valid starting point for analyzing the cortical network's function and grasping the basic mechanism for direction selectivity, thus providing a possible alternative to 'spiking -models' (Maex and Orban 1996; Suarez et al. 1995). A progressive (i.e., hierarchical) complication of the model, e.g., by collecting the recurrent loop activity on higher layers, would account for the observed variability in the spatiotemporal responses of cortical simple cells, without compromising the modus operandi of the basic architectural core. Further improvements in the model should consider a number of other phenomena that might contribute to direction selectivity, in particular, the effect of a combination of lagged and nonlagged geniculate inputs (Mastronarde 1987; Saul and Humphrey 1990; Wimbauer et al. 1994, 1997), expansive nonlinearities (Emerson 1997; Heeger 1993), and ON-OFF interactions (Ferster 1988; Gaudiano 1994). We plan to investigate these topics in future studies.

Acknowledgements. We wish to thank Prof. Giacomo M. Bisio for stimulating discussions and helpful advice. This work was partially supported by the CEC-Esprit BR Project CORMORANT-8503, by the University of Genoa Project 'Strutture Fisiche per la Percezione e l'Elaborazione', and by the ASI Project 'Microsistemi Ottici per la Robotica Spaziale'.

\section{References}

Adelson EH, Bergen JR (1985) Spatiotemporal energy models for the perception of motion. J Opt Soc Am 2:284-321

Adelson EH, Bergen JR (1991) The plenoptic function and the elements of early vision. In: Landy MS, Movshon JA (eds) Computational models of visual processing. MIT Press, Cambridge, Mass, pp 3-20

Albus K (1975) A quantitative study of the projection area of the central and the paracentral visual field in area 17 of the cat. Exp Brain Res 24:159-202

Amari S (1977) Dynamics of pattern formation in lateral-inhibition type neural fields. Biol Cybern 27:77-87

Arieli A, Shoham D, Hildesheim R, Grinvald A (1995) Coherent spatiotemporal patterns of ongoing activity revealed by realtime optical imaging coupled with single-unit recordings in the cat visual cortex. J Neurophysiol 73:2072-2093

Carandini M, Heeger DJ (1994) Summation and division by neurons in primate visual cortex. Science 264:1333-1336

De Angelis GC, Ohzawa I, Freeman RD (1993a) Spatiotemporal organization of simple-cell receptive fields in the cat's striate cortex. I. General characteristics and postnatal development. J. Neurophysiol 69:1091-1117

De Angelis GC, Ohzawa I, Freeman RD (1993b) Spatiotemporal organization of simple-cell receptive fields in the cat's striate cortex. II. Linearity of temporal and spatial summation. J Neurophysiol 69:1118-1135

De Angelis GC, Ohzawa I, Freeman RD (1995) Receptive-field dynamics in the central visual pathways. Trends Neurosci 18:451-458

Douglas RJ, Martin KAC, Whitteridge D (1989) A canonical microcircuit for neocortex. Neural Comp 1:480-488

Douglas RJ, Koch C, Mahowald M, Martin KAC, Suarez HH (1995) Recurrent excitation in neocortical circuits. Science 269:981-985

Eckhorn R, Krause F, Nelson JI (1993) The RF-cinematogram. Biol Cybern pp. 37-55

Emerson RC (1997) Quadrature subunits in directionally selective simple cells: spatiotemporal interactions. Visual Neurosci 14:357-371

Emerson RC, Huang MC (1997) Quadrature subunits in directionally selective simple cells: counterphase and drifting grating responses. Visual Neurosci 14:373-385

Ferster D (1988) Spatially opponent excitation and inhibition in simple cells of the cat visual cortex. J Neurosci 8:11721180

Ganz L (1984) Visual cortical mechanisms responsible for direction selectivity. Vision Res 24:3-11

Gaudiano P (1994) Simulations of X and Y retinal ganglion cell behavior with a nonlinear push-pull model of spatiotemporal retinal processing. Vision Res 34:1767-1784

Grinvald A, Lieke EE, Frostig RD, Hildesheim R (1994) Cortical point-spread function and long-range lateral interactions revealed by real-time optical imaging of macaque monkey primary visual cortex. J Neurosci 14:2545-2568

Grossberg S (1976) Adaptive pattern classification and universal recoding. I. Parallel development and coding of neural feature detectors. Biol Cybern 23:121-134

Hamilton DB, Albrecht DG, Geisler WS (1989) Visual cortical receptive fields in monkey and cat: spatial and temporal phase transfer function. Vision Res 29:1285-1308

Heeger DJ (1993) Modeling simple-cell direction selectivity with normalized, half-squared, linear operators. J Neurophysiol 70:1885-1898

Jones J, Palmer L (1987) The two-dimensional spatial structure of simple receptive fields in cat striate cortex. $\mathbf{J}$ Neurophysiol 58:1187-1211

Koch C, Hildreth EC (1987) The analysis of visual motion: from computational theory to neuronal mechanism. Annu Rev Neurosci 10:477-533 
Lohmann H, Eckhorn R, Reitboeck HJ (1988) Visual receptive fields of local intracortical potentials. J Neurosci Methods 25:29-44

Luhmann HJ, Greuel JM, Singer W (1990a) Horizontal interactions in cat striate cortex. II. A current source-density analysis. Eur J Neurosci 2:358-368

Luhmann HJ, Greuel JM, Singer W (1990b) Horizontal interactions in cat striate cortex. III. Ectopic receptive fields and transient exuberance of tangential interactions. Eur J Neurosci $2: 369-377$

Maex R, Orban GA (1992) A model circuit for cortical temporal low-pass filtering. Neural Comp 4:932-945

Maex R, Orban GA (1996) Model circuit of spiking neurons generating directional selectivity in simple cells. J Neurophysiol $75: 1515-1545$

Mallot HA (1995) Layered computation in neural networks. In Arbib MA (ed) The handbook of brain theory and neural networks, MIT Press, Cambridge, Mass., p 513-516.

Mallot HA, Giannakopoulos F (1996) Population networks: a large-scale framework for modelling cortical neural networks. Biol Cybern 75:441-452

Mallot HA, Seelen W von, Giannakopoulos F (1990) Neural mapping and space variant image processing. Neural Networks $3: 245-263$

Malsburg von der Ch (1973) Sel-organization of orientation selective cells in the striate cortex. Kybernetik 32:49-62

Mastronarde DN (1987) Two classes of single-input x-cells in cat lateral geniculate nucleus. I. Receptive field properties and classification of cells. J Neurophysiol 57:357-380

McLean J, Palmer LA (1989) Contribution of linear spatiotemporal receptive field structure to velocity selectivity of simple cells in area 17 of cat. Vision Res 29:675-679

McLean J, Raab S, Palmer LA (1994) Contribution of linear mechanisms to the specification of local motion by simple cells in areas 17 and 18. Visual Neurosci 11:271-294

Nicholson C (1973) Theoretical analysis of field potential in anisotropic ensembles of neuronal elements. IEEE Trans Biomed Eng 20:278-288

Orban GA (1984) Neuronal operations in the visual cortex. In: Studies of brain functions. Springer, Berlin Heidelberg New York

Reid RC, Soodak RE, Shapley RM (1991) Directional selectivity and spatiotemporal structure of receptive fields of simple cells in cat striate cortex. J Neurophysiol 66:505-529

Ruff PI, Rauschecker JP, Palm G (1987) A model of directionselective 'simple' cells in the visual cortex based on inhibition asymmetry. Biol Cybern 57:147-157

Sabatini SP (1996) Recurrent inhibition and clustered connectivity as a basis for Gabor-like receptive fields in the visual cortex. Biol Cybern 74:189-202
Sabatini SP, Solari F, Bisio GM (1996) An architectural mechanism for direction-tuned cortical simple cells: the role of mutual inhibition. In Proc NIPS'96, Denver, December

Saul AB, Humphrey AL (1990) Spatial and temporal response properties of lagged and non-lagged cells in the cat lateral geniculate nucleus. J Neurophysiol 64:206-224

Seelen von W, Mallot HA, Giannakopoulos F (1987) Characteristics of neuronal systems in the visual cortex. Biol Cybern 56:37-49

Sillito AM (1977) Inhibitory mechanisms underlying the directional selectivity of simple, complex and hypercomplex cells in the cat's visual cortex. J Physiol 271:699-720

Somers DC, Nelson SB, Sur M (1995) An emergent model of orientation selectivity in cat visual cortical simple cells. J Neurosci 15:5448-5465

Stratford KJ, Tarczy-Hornoch K, Martin KAC, Bannister NJ, Jack JJB (1996) Excitatory synaptic inputs to spiny stellate cells in cat visual cortex. Nature 382:258-261

Suarez H, Koch C, Douglas R (1995) Modeling direction selectivity of simple cells in striate visual cortex within the framework of the canonical microcircuit. J Neurosci 15:6700-6719

Tolhurst DJ, Dean AF (1991) Evaluation of a linear model of directional selectivity in simple cells of the cat's striate cortex. Visual Neurosci 6:421-428

Watson AB, Ahumada AJ (1985) Model of human visual-motion sensing. J Opt Soc Am 2:322-341

Wehmeier U, Dong D, Koch D, Van Essen D (1989) Modeling the mammalian visual system. In: Methods in neural modeling. MIT Press, Cambridge, Mass

Wilson HR (1985) A model for direction selectivity in threshold motion perception. Biol Cybern 51:213-222

Wilson HR, Cowan JD (1972) Excitatory and inhibitory interactions in localized populations of model neurons. Biophys $\mathbf{J}$ 12:1-23

Wimbauer S, Gerstner W, Hemmen JL van (1994) Emergence of spatiotemporal receptive fields and its application to motion detection. Biol Cybern 72:81-92

Wimbauer S, Wenisch OG, Hemmen JL van, Miller KD (1997) Development of spatiotemporal receptive fields of simple cell. II. Simulation and analysis. Biol Cybern 77:463-477

Wörgötter F, Holt F (1991) Spatiotemporal mechanisms in receptive fields of visual cortical simple cells: a model. J Neurophysiol 65:494-510

Wörgötter F, Koch C (1991) A detailed model of the primary vision pathway in the cat: comparison of afferrent excitatory and intracortical inhibitory connection schemes for orientation selectivity. J Neurosci 11:1959-1979

Xing J, Gerstein GL (1996) Networks with lateral connectivity. I. Dynamic properties mediated by the balance of intrinsic excitation and inhibition. J Neurophysiol 75:184-199 